\title{
Determinação de hidrocarbonetos em sedimentos de canais de drenagem pluvial Pelotas/RS
}

A qualidade das águas superficiais, de infiltração e águas subterrâneas é influenciada pelos poluentes coletados das superfícies impermeabilizadas e transportados pelo escoamento das águas pluviais urbanas (runoff). Dentre os constituintes do runoff urbano os hidrocarbonetos são considerados indicadores geoquímicos e podem inferir sobre as diferentes fontes de contaminação. Estes compostos são facilmente adsorvidos ao material particulado sendo acumulado nos sedimentos que atuam como um reservatório para contaminantes hidrofóbicos. O objetivo deste trabalho foi avaliar o impacto das cargas de hidrocarbonetos transportadas pelo runoff urbano. Desta forma, estudou-se um canal de drenagem pluvial que atravessa a área urbana de Pelotas/RS, avaliando os níveis de n-alcanos, HTP (hidrocarbonetos totais de petróleo), Alifáticos, Misturas Complexas Não Resolvidas (MCNR) e suas relações com as fontes de contaminação. Os valores HTP variaram entre 4,2 × $105 \mu \mathrm{g} \mathrm{kg-1} \mathrm{a} \mathrm{9,9} \mathrm{\times} 105 \mu \mathrm{g} \mathrm{kg-1.} \mathrm{O} \mathrm{teor} \mathrm{alifático} \mathrm{total} \mathrm{indicou} \mathrm{contaminação} \mathrm{crônica.} \mathrm{O} \mathrm{estudo} \mathrm{comprovou} \mathrm{a} \mathrm{predominância} \mathrm{de} \mathrm{n-alcanos} \mathrm{entre}$ C23 e C35 em todos os pontos. O total de n-alcanos variou de 1,8 × 104 a 7,8 x $104 \mu \mathrm{g} \mathrm{kg-1.} \mathrm{Os} \mathrm{resultados} \mathrm{apontaram} \mathrm{para} \mathrm{fontes} \mathrm{mistas} \mathrm{de} \mathrm{contaminação.} \mathrm{Os}$ níveis de hidrocarbonetos (HTP, alifáticos e $n$-alcanos) foram considerados elevados, confirmando o impacto do escoamento urbano no sistema de drenagem da cidade e seu consequente impacto nos recursos hídricos.

Palavras-chave: n Alcanos; Poluição Ambiental; Runnof.

\section{Determination of hydrocarbons in sediments from rain drainage channels Pelotas/RS}

\begin{abstract}
The quality of surface water, infiltration and groundwater is influenced by pollutants collected from waterproofed surfaces and transported by urban runoff. Among the constituents of the urban runoff hydrocarbons are considered as geochemical indicators and can infer about the different sources of contamination. These compounds are readily adsorbed to the particulate material being accumulated in the sediments that act as a reservoir for hydrophobic contaminants. The objective of this work was to evaluate the impact of the hydrocarbon cargo transported by the urban runoff. In this way, a drainage channel was studied that crosses the urban area of Pelotas/RS, evaluating the levels of $n$-alkanes, TPH (total petroleum hydrocarbons) Aliphatic, Unresolved Complex Mixtures (UCM) and their connections with sources of contamination. TPH values ranged from $4.2 \times 105 \mu \mathrm{gg}-1$ to $9.8 \times 105 \mu \mathrm{g} \mathrm{kg}-1$. Total aliphatic content indicated chronic contamination. Samples showed predominance of C23 to C35 n-alkanes at all points, ranging from $1.8 \times 104$ to $7.8 \times 104 \mu \mathrm{g} \mathrm{kg}-1$. The results pointed to mixed sources of contamination Hydrocarbon levels (HTP, aliphatic and n-alkanes) were considered high, confirming the impact of urban drainage on the city's drainage system and its consequent impact on water resources.
\end{abstract}

Keywords: n Alkanes; Environmental Pollution; Runnof.

Topic: Química Agrícola e Ambiental

Reviewed anonymously in the process of blind peer.
Received: $10 / 02 / 2018$

Approved: 24/03/2018
Pedro José Sanches Filho (iD

Instituto Federal Sul-Rio-Grandense, Brasil

http://lattes.cnpq.br/9785390634457316

http://orcid.org/0000-0002-9852-8940

pjsans@ibest.com.br

Gisseli Oliveira de Mesquita

Instituto Federal Sul-Rio-Grandense, Brasil

go-montenegro@bol.com.br
Referencing this:

SANCHES FILHO, P. J.; MESQUITA, G. O.. Determinação de hidrocarbonetos em sedimentos de canais de drenagem pluvial Pelotas/RS. Revista Ibero Americana de Ciências Ambientais, v.9, n.3, p.38-49, 2018. DOI: http://doi.org/10.6008/CBPC21796858.2018.003.0004 


\section{INTRODUÇÃO}

Durante os eventos de precipitação pluviométrica, a água da chuva lava o material particulado da atmosfera e superfícies urbanas impermeabilizadas, consequentemente, compostos dissolvidos, coloidais, partículas sólidas são liberadas em uma mistura heterogênea transportada pelo escoamento das águas das chuvas captadas pelos canais de drenagem pluvial e conduzidas a rios e mares. Esta água de escoamento superficial é chamada de runoff. Esta mistura inclui compostos orgânicos, inorgânicos, nutrientes, óleos, graxas e metais pesados (WU et al., 2001; AZEVEDO et al., 2002; MILLE et al., 2007). Segundo Gnecco et al., (2005) a carga de poluente associada ao runoff é produzida principalmente pelo tráfego de veículos e outras atividades humanas.

A qualidade das águas superficiais, infiltrações e águas subterrâneas é influenciada por poluentes coletados de superfícies impermeáveis e transportados pelo escoamento das águas pluviais urbanas (GÖBEL et al., 2007). A mistura de poluentes encontrados na água das chuvas varia de acordo com a captação, o uso da terra e a exposição da água a várias superfícies (BARBOSA et al., 2012). 0 tratamento das águas pluviais é um problema crítico, devido às suas altas cargas de contaminantes. Vários poluentes prioritários considerados pela EPA( Environmental Protection Agency) foram identificados em águas pluviais (ERIKSSON et al., 2007), dentre estes compostos destacam-se os hidrocarbonetos (HC).

O aumento da atenção científica tem sido dedicado à obtenção de informações sobre marcadores químicos naturais e antrópicos. Os HC são compostos naturais e componentes importantes da matéria orgânica sedimentar de diferentes ambientes hídricos, como rios, lagos, zonas húmidas e áreas costeiras marinhas (ANDERSSON et al., 2014; HOWITT et al., 2014; SANCHES FILHO et al., 2013; SANCHES FILHO et al., 2012). A sua composição qualitativa e quantitativa é significativamente afetada pelas atividades humanas. São misturas complexas provenientes de fontes biogênicas, diagênicas, petrogênicas e pirogênicas (WU et al.,2001; AZEVEDO et al., 2002; MILLE et al., 2007).

Estes compostos são facilmente adsorvidos em material particulado acumulamdo-se nos sedimentos do fundo que atuam como um reservatório para contaminantes hidrofóbicos (ANDERSSON, 2014). A constituição qualitativa e quantitativa do HC e alguns tipos de índices podem ser usado para identificar fontes de hidrocarbonetos como biogênicas (terrestres ou aquáticas) e/ou antropogênicas (COMMENDATORE et al., 2012).

Entre os HC biogênicos, os $n$-alcanos são predominantes em plantas e animais (NISHIJIMA et al., 2001). $n$-alcanos biogênicos são tipicamente caracterizados por séries homólogas de $n$-alcanos de cadeia curta (nC15 - nC19) derivados de algas ou n-alcanos de cadeia longa (nC25 - nC35); derivados de ceras epicuticulares de plantas superiores, que são relacionadas com uma forte predominância de números de carbono ímpares sobre pares (YUNKER et al., 1993). Os hidrocarbonetos alifáticos também são os principais componentes dos produtos petrolíferos, que produzem uma distribuição de $n$-alcanos em que a proporção de cadeia de carbono ímpares e pares são aproximadamente iguais em abundância (produzindo um índice de preferência de carbono [IPC] de $\approx 1$. Muitas vezes encontram-se associados a misturas complexas não 
resolvidas (MCNR) constituidas por diversos hidrocarbonetos como alcanos ramificados e cíclicos (COLOMBO et al., 1989; MARTINS et al., 2004; SIMPSON et al., 2005).

Os hidrocarbonetos aromáticos policíclicos (HPAs) derivam principalmente de fontes antropogênicas, como a combustão de combustíveis fósseis e a liberação direta de petróleo e produtos petrolíferos, além de uma pequena contribuição resultante de incêndios florestais e runoff agrícola (AZEVEDO et al., 2002; VOLKMAN et al., 1992). Os HPAs raramente são encontrados como produtos biossintéticos (COMMENDATORE et al., 2012) e possuem alta toxicidade em organismos devido ao seu potencial cancerígeno e mutagênico (NISHIGIMA et al., 2001; LI et al., 2008). A presença de HPAs e uma composição de mistura em sedimentos também podem fornecer indicação das fontes de poluição (COMMENDATORE et al., 2012). A Agência de Proteção Ambiental dos Estados Unidos (USEPA) e a Comunidade Européia (UE) lista HPA's como poluentes prioritários (BOULOUBASSI et al., 2001).

A cidade de Pelotas/RS no Brasil está localizada nas margens da Lagoa Patos (sul do Brasil). A região conhecida como 'Saco do Laranjal' tem uma grande importância econômica devido ao seu turismo ambiental em que funciona como viveiro para muitas espécies de peixes e crustáceos. A área é limitada pelo 'Ponta da Feitoria' (norte) e o 'Canal São Gonçalo' (sul), que faz parte da área estuarina da Lagoa dos Patos. O Canal São Gonçalo recebe o impacto direto da cidade do escoamento gerado pela área urbana da cidade de Pelotas e transportado por canais de drenagem de águas pluviais (Figura 1).

Este canal, em particular, representa o ponto de transporte de grande quantidade de contaminantes para área. Recebe efluentes industriais, esgoto doméstico e toda a água coletada do sistema de drenagem de águas pluviais, para avaliar o efeito da cidade neste sistema, foi escolhido um canal de drenagem de águas pluviais que atravessa diferentes áreas urbanas da cidade de Pelotas e recebe escoamento de diferentes regiões. Há pouca informação sobre este tipo de contaminação, pelo que é extremamente importante avaliar um tratamento adequado para esta fonte difusa de poluição, ou seja, o runnof. Assim, o objetivo deste estudo é avaliar possíveis fontes de hidrocarbonetos, que são derivadas de áreas urbanas e coletadas nos sistemas de drenagem de águas pluviais, e também determinar os níveis de $n$-alcanos no canal estudado.

\section{MATERIAIS E MÉTODOS}

Para avaliar os níveis de contaminação e as possíveis fontes de HC foi selecionado o canal de drenagem pluvial no prolongamento da avenida São Francisco de Paula que atravessa vários bairros na área urbana da cidade de Pelotas/RS/Brasil, foram coletadas cinco amostras de sedimentos em Setembro de 2013 nos locais apresentados na Figura 1. A posição exata para cada ponto de amostragem (Fig.1) foi definida por medições com GPS (Sistema de Posicionamento Global) Garmin \& $\operatorname{Trex}^{\circledR}$, precisão de $\pm 15 \mathrm{~m}$.

Este canal recebe ao longo do seu escoamento cursos de águas locais como estacionamento, ruas pavimentadas e áreas rurais próximas de postos de gasolina. Também é possível que os fluxos ilegais de esgotos doméstico sejam recebidos. Caracterização dos pontos de amostragem (localização) - Conforme descrito por GRIMMLER et al. (2016). Ponto 1 (P1): No entroncamento de duas avenidas pavimentas com fluxo intenso de veículos automotores, próximo a um posto de gasolina; Ponto 2 (P2): Ao lado do shopping 
Center no escoamento das águas do seu estacionamento no canal; Ponto 3 (P3): Situado nas proximidades de um condomínio residencial em construção, em uma zona menos urbanizada; Ponto 4 (P4): Situado no aporte de um canal afluente que atravessa áreas urbanizadas e com denso tráfego de veículos, junto ao canal em estudo; Ponto 5 (P5): É o encontro do canal com um outro canal de drenagem que atravessa uma área rural com baixa contribuições antropogênicas, situado próximo ao canal São Gonçalo.
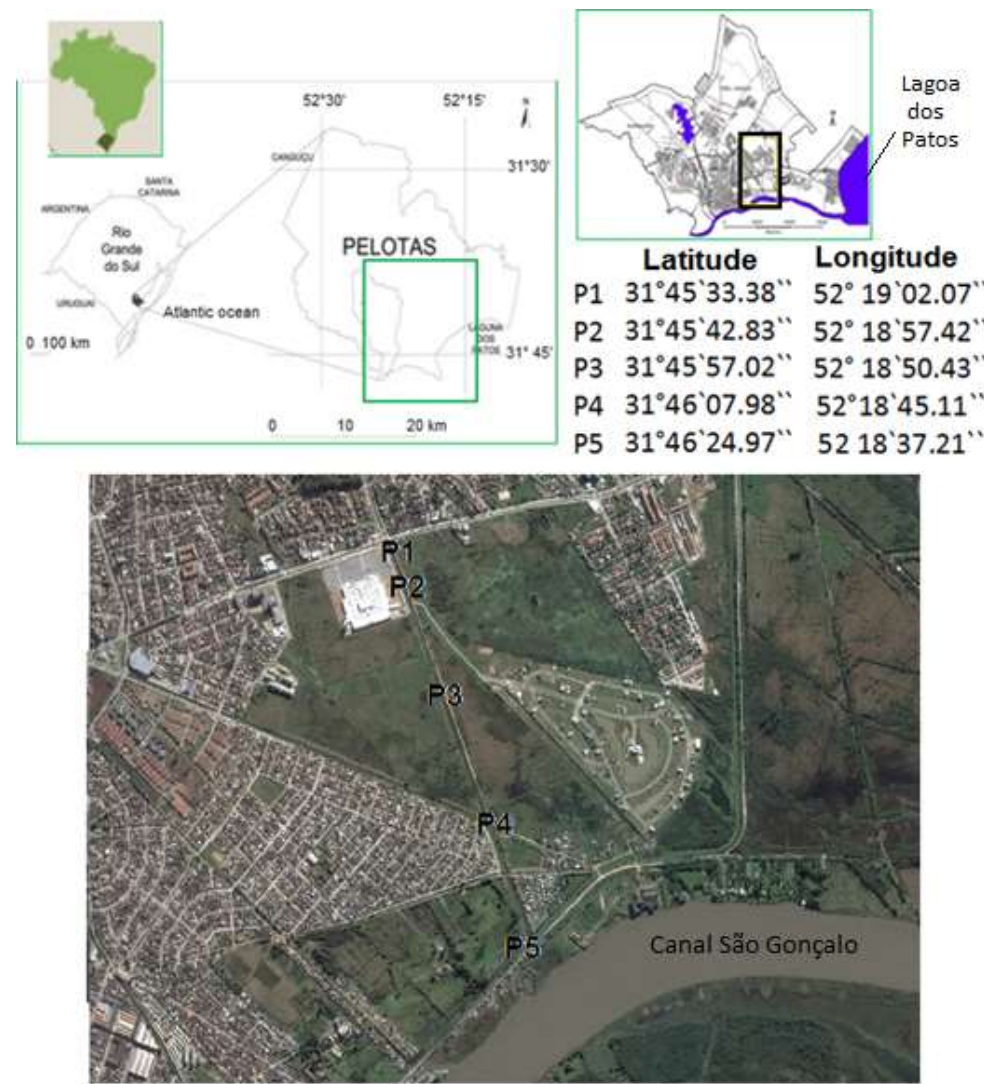

Figura 1: Localização do ponto de coleta-modificado de Grimmler et al. (2016).

Os sedimentos ( 0 a $5 \mathrm{~cm}$ de profundidade) foram coletados utilizando uma draga de aço inoxidável do tipo Van Veen. As amostras foram colocadas em frascos de vidro previamente limpos com solvente (hexano e diclorometano) envoltos em papel alumínio. Todas as amostras foram homogeneizadas e imediatamente resfriadas após a coleta a $4^{\circ} \mathrm{C}$ até análises laboratoriais.

Os sedimentos coletados foram homogeneizados e sub-amostrados para determinação da umidade, matéria orgânica total e distribuição granulométrica. O teor de umidade foi obtido por método gravimétrico descrito por (TEDESCO et al., 1995). A matéria orgânica total (MOT) foi determinada pelo método de calcinação (BISSUTTI et al., 2004; MANNINO et al., 2008) e convertida em carbono orgânico total (COT) utilizando a fórmula MOT (\%) =1,724 × COT (fator Van Bemmelen). A granulometria foi realizada pelo método padrão de peneiras (SUGUIO et al., 1973). O nitrogênio orgânico total (NOT) foi determinado pelo método de Kjeldahl (APRILE et al., 2003). Solventes (diclorometano - DCM, n-hexano-hex e acetona grau P.A), sulfato de sódio anidro e sílica gel 60 (70-230 mesh ASTM) foram obtidos pela Merck (Darmstadt, Alemanha); A alumina neutra (tipo 507, ASTM 70-290 mesh) foi obtida da Fluka AG (Suíça) e os cartuchos de extração de celulose (20 mm i.d. e $80 \mathrm{~mm}$ de comprimento) foram obtidos de Whatman (Reino Unido). A sílica, a alumina 
e o sulfato de sódio anidro foram ativados a 400 C durante 4 horas. As folhas de alumínio foram limpas com acetona e secas à temperatura ambiente antes do uso.

As agulhas de cobre (Sigma-Aldrich, EUA) foram ativadas com HCl concentrado e foram ainda lavadas com água purificada (duas vezes), metanol (duas vezes), DCM (duas vezes) e hexano (duas vezes) e depois armazenadas em hexano. Padrões cromatográficos de $n$-alcanos (C8 a C20 e C21 a C40) foram adquiridos a Sigma Aldrich (EUA). A solução para calibração foram obtidas por diluição da mistura padrão de $40 \mathrm{mg} \mathrm{L}^{-1} \mathrm{em}$ diclorometano.

Para determinar o desempenho da extração, fortificou-se uma massa de $20 \mathrm{~g}$ de sedimento com 100 $\mu \mathrm{L}$ do padrão de $n$-alcanos pares (C10 a C40) em concentração de $50 \mathrm{mg}$ L-$^{1}$ em $n$-heptano (de Fluka Analytical). Para o cálculo da recuperação, a concentração de $n$-alcanos fortificada foi descontada da concentração $n$-alcanos presentes no sedimento sem fortificação.Os limites de detecção (LD) e quantificação (LQ) foram realizados a partir de leituras do branco da amostra no cromatógrafo, que foram preparados nas mesmas condições das amostras. O LQ foi calculado como sendo a média do branco mais dez vezes o desvio padrão e LD como média do branco mais três vezes o desvio padrão do branco (IUPAC, 1997). Todos os materiais utilizados foram previamente descontaminados com detergente e água destilada, seguidos de enxágues com acetona, hexano e diclorometano, com posterior secagem. Os solventes utilizados foram previamente destilados e os procedimentos analíticos foram realizados em triplicata.

\section{Extração e fracionamento}

A extração dos analitos de $20 \mathrm{~g}$ de amostra úmida foi feita em um banho ultra-sônico. Utilizou-se como solvente uma mistura de hexano e acetona 1:1 por 3 ciclos de 30 minutos a cada ciclo de extração o solvente foi separado por filtração, seguindo o método USEPA 3550B. Os extratos passaram por uma coluna cheia de $\mathrm{Na}_{2} \mathrm{SO}_{4}$ anidro. $\mathrm{O}$ filtrado recolhido foi reduzido até aproximadamente $10 \mathrm{ml}$ num evaporador rotativo, o volume foi transferido para béquer e mantido em capela, até secagem completa. Agulhas de cobre ativadas foram adicionadas durante a extração para remoção de enxofre (ZHENG et al., 1999), a fim de evitar qualquer interferência durante a análise por cromatografia gasosa acoplada ao espectrometro de massas (GCMS).

O fracionamento dos extratos foi realizado utilizando uma coluna com $1 \mathrm{~g}$ de $\mathrm{Na}_{2} \mathrm{SO}_{4}$ anidro, 3,2g de sílica $\left(\mathrm{SiO}_{2}\right)$ - Desativado a $5 \%, 1,8 \mathrm{~g}$ de alumina $\left(\mathrm{Al}_{2} \mathrm{O}_{3}\right)$ - $2 \%$ desativado e $1 \mathrm{~g}$ de $\mathrm{Na}_{2} \mathrm{SO}_{4}$ anidro na parte superior. Os extratos foram misturados com $0,5 \mathrm{~g}$ de sílica e adicionados no topo de uma coluna. A sequência de solventes para eluição foi de $20 \mathrm{~mL}$ de $\mathrm{n}$-hexano (fração F1 - hidrocarbonetos alifáticos), $20 \mathrm{~mL}$ de solução de $\operatorname{DCM}$ / n-hexano (1:5 v / v) e $20 \mathrm{~mL}$ de solução de DCM / hexano (1:1v / v) que foram reunidas na fração F2 (compostos poliaromáticos (UNEP, 1992). Todas as frações foram evaporadas e controladas gravimétricamente. A seguir foram avolumadas a $1 \mathrm{ml}$ em vial e armazenadas a $-4^{\circ} \mathrm{C}$. As frações foram monitoradas gravimetricamente, sendo que a fração de massa F1 resultou no conteúdo de alifáticos e a soma das frações 1 e 2 resultou no conteúdo de hidrocarbonetos totais de Petóleo (HTP) (SANCHES FILHO et al., 2017). 
Para análise cromatográfica, a fração (F1) foi reconstituída em DCM ( $1 \mathrm{~mL}$ ) e uma alíquota de $1 \mu \mathrm{L}$ para cada extrato foi injetada no GCMS em Splitless, sendo analisada em modo SCAN. Os hidrocarbonetos foram quantificados usando um GCMS (Shimadzu QP 2010 ultra) com uma coluna RTX 5MS (30m x 0.25mm i.d. $\times 0.25 \mu \mathrm{m}$ de espessura de filme). $O$ injetor e a interface foram mantidos a $280^{\circ} \mathrm{C}$ e fonte de íons em $200^{\circ} \mathrm{C}$, mas a curva de calibração para os hidrocarbonetos n-alcanos lineares nC9 a nC40 abrangeu um intervalo entre 0,2 e 4,0 $\mathrm{mg} \mathrm{L}^{-1}$ com cinco pontos de diferentes concentrações. Cada ponto da curva de calibração representa o valor médio de três áreas de medidas independentes. $O$ índice de preferência de carbono (IPC) Celino et al. (2008) foi usado para detectar a contribuição da ceras vegetais versus contaminação por combustível fóssil. O IPC foi calculado da seguinte forma:

$$
I P C=\frac{1}{2}\left[\frac{\mathrm{C} 25+\mathrm{C} 27+\mathrm{C} 29+\mathrm{C} 31+\mathrm{C} 33}{\mathrm{C} 24+\mathrm{C} 26+\mathrm{C} 28+\mathrm{C} 30+\mathrm{C} 32}\right]+\left[\frac{\mathrm{C} 25+\mathrm{C} 27+\mathrm{C} 29+\mathrm{C} 31+\mathrm{C} 33}{\mathrm{C} 26+\mathrm{C} 28+\mathrm{C} 30+\mathrm{C} 32+\mathrm{C} 34}\right]
$$

A razão terrígena / aquática (RTA) (MILLE et al., 2007), que é a relação entre as concentrações de nalcanos de cadeia longa (nC27 + nC29 + nC31) versus n-alcanos de cadeia curta (nC15 + nC17 + nC19), avalia a prevalencia de fontes biogênica como terrestre ou aquáticas (MILLE et al., 2007; YUNKER et al., 1993); APRILE et al., 2003). As concentrações de MCNR(Mistura Complexa não Resolvida) foram avaliadas segundo método EPA 8015B conforme a equação multiplicando-se a àrea da MCNR por um fator de resposta médio calculado a partir dos padrões de n-alcanos. Para cálculos de correlação de Pearson foi utilizado o Excel 2010.

\section{RESULTADOS E DISCUSSÕES}

A Tabela 1 apresenta os resultados para caracterização preliminar dos sedimentos, bem como, os níveis de Alifáticos e HTP.

Tabela 1: Caracterização preliminar dos sedimentos nos pontos do Canal São Fracisco de Paula.

\begin{tabular}{|l|l|l|l|l|l|l|l|l|}
\hline Pontos & $\begin{array}{l}\text { MOT\% } \\
\text { RSD\% }\end{array}$ & $<63 \mu \mathrm{m}$ & UMIDADE\% \pm RSD\% & COT\% & $\begin{array}{l}\text { NOT\% } \\
\text { RSD\% }\end{array}$ & $\begin{array}{l}\text { ALIFÁTICO }\left(10^{5} \mu \mathrm{g}\right. \\
\text { Kg) } \pm \% R S D\end{array}$ & COT/NOT & $\begin{array}{l}\text { HTP } \\
\pm \% R S D\end{array}$ \\
\hline P1 & $2,1 \pm 14,8$ & 0,2 & $23,2 \pm 0,3$ & 1,21 & $0,02 \pm 28,2$ & $2,8 \pm 9,4$ & 48,4 & $4,2 \pm 8,3$ \\
\hline P2 & $11,2 \pm 6,1$ & 8,5 & $60,4 \pm 10,5$ & 6,5 & $0,09 \pm 11,0$ & $3,6 \pm 4,9$ & 72,2 & $6,3 \pm 2,9$ \\
\hline P3 & $4,8 \pm 22,6$ & 4,6 & $39,7 \pm 4,6$ & 2,8 & $0,09 \pm 6,1$ & $3,6 \pm 18,8$ & 31,1 & $5,1 \pm 16,2$ \\
\hline P4 & $13,7 \pm 2,4$ & 10,3 & $74,4 \pm 0,2$ & 8,0 & $0,08 \pm 18,3$ & $5,9 \pm 2,2$ & 96,4 & $9,8 \pm 2,6$ \\
\hline P5 & $3,7 \pm 17,4$ & 9,4 & $38,6 \pm 17,2$ & 2,2 & $0,04 \pm 0$ & $2,5 \pm 3,1$ & 55,0 & $4,5 \pm 8,6$ \\
\hline
\end{tabular}

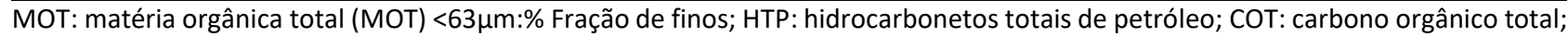
NOT: Nitrogênio orgânico total; \% RSD: desvio padrão relativo em\%.

Podemos observar a partir dos dados que os pontos 2 e 4 apresentam os maiores percentuais de finos $(<63 \mu \mathrm{m})$. A granulometria do sedimento é essencial para a acumulação e persistência de hidrocarbonetos no ambiente. O baixo tamanho de partícula é responsável por uma elevada capacidade de adsorção de sedimentos (VIÑAS et al., 2009). Este resultado está em acordo com os níveis de MOT e umidade. A baixa granulometria aumenta os espaços intersticiais que ficam preenchidos pela água nos sedimentos.

A matéria orgânica total dos sedimentos neste estudo variou de $2,1 \% \pm 14,8 \%$ no ponto 1 , para $13,7 \%$ $\pm 2,4 \%$ no ponto 4 . Os pontos de amostragem 2 e 4 exibiram um sedimento rico em matéria orgânica. Valores acima de $10 \%$ são caracterizadas como sedimentos de origem orgânica. Estes resultados apontam para os pontos 2 e 4 como locais com condições hidrodinâmicas lênticos que favorecem a deposição e acúmulo de contaminantes. 
Os níveis de do HTP e de hidrocarbonetos alifáticos seguiram a mesma distribuição da MOT concentrando-se nos sedimentos dos pontos 2 e 4, confirmando que esses pontos são lugares potenciais para a acumulo de poluentes como HPAs, n-alcanos e outros. Grimmler et al., (2015) em seu estudo de metais tóxicos no mesmo canal de drenagem pluvial detectou nestes pontos os maiores niveis de $\mathrm{Cr}, \mathrm{Cu}, \mathrm{Zn}$ e $\mathrm{Pb}$.

Valores superiores a $3 \times 10^{5} \mu \mathrm{g} \mathrm{kg}^{-1}$ para HTP e superiores a $5 \times 10^{5} \mathrm{\mu g} \mathrm{kg}^{-1}$ para alifáticos, indicam sedimentos poluídos por hidrocarbonetos (VOLKMAN et al., 1980). Os valores de HTP indicam altos níveis de contaminação orgânica em todos os pontos, refletindo a alta carga de hidrocarbonetos recebida e transportada pelo canal de drenagem pluvial estudado. No ponto 4, os níveis de concentração alifática foram $593.8585 \mu \mathrm{g} \mathrm{kg}^{-1} \pm 2,2 \%$, o que confirma a contaminação por hidrocarbonetos também através deste parâmetro. Neste ponto, o canal recebe carga de outro canal de drenagem pluvial, que drena uma área da cidade densamente urbanizada. A entrada deste tributário, elevou os níveis de MOT e quase duplicou as concentrações de HTP e alifáticas. Esta situação confirma a influência da zona urbana na carga de poluntes gerada e transportada para pelos canais de drenagem pluvial. Outro parâmetro utilzado para avaliação do tipo de fonte de contaminação é relação COT/NOT. Neste estudo os valores variaram entre 31,1 a 96,4; as razões $>20$ indicaram uma matéria orgânica originada de planta terrestre superiores (SAITO et al., 1989). Os coeficientes de correlação de Pearson entre Finos e MOT foram de 0,70; entre Finos e umidade foi de 0,80; e entre Finos e HTP foi de 0,92.

\section{Determinação de $n$-alcanos}

Os resultados da análise cromatográfica de n-alcanos podem ser observados na Figura 2 e Tabelas 2 e 3, sendo que na Tabela 2 apresenta as Figuras de mérito para o método anlítico. Observa-se que o método cromatográfico mostrou uma resposta linear, para todos os $n$-alcanos estudados, no intervalo de concentração entre 0,2 a 4,0 mg L-1, com coeficientes de correlação linear $\left(r^{2}\right)$ que variam de 0,985 a 0,999; Os limites de detecção (LD) e os limites de quantificação (LQ) variaram entre 1,5 e 14,2 $\mu \mathrm{kg}^{-1}$ e 2,1 a 22,0 $\mu \mathrm{g} \mathrm{kg}^{-1}$, respectivamente (considerando uma recuperação de $100 \%$ e uma massa seca de $10 \mathrm{~g}$ ). Os valores mais elevados de LD e LQ estão relacionados à redução do fator de resposta para compostos mais pesados, como na faixa de C35 a C40. As recuperações para os $n$-alcanos variam de $36,37 \% \pm 10,78 \%$ para $C 10$ a $68,4 \%$ $\pm 4,9 \%$ para C14 e 70,0\% $\pm 7,2 \%$ a 85,9\% $\pm 7,1 \%$ para a faixa de $\mathrm{C} 16$ a C40, não sendo necessário corrigir os valores de $n$-alcanos encontradas nas amostras. Os valores encontrados para C10 a C14 estão relacionados a perdas por volatilização em etapas de redução de volume.

Tabela 2: Figuras de mérito para o método de determinação para os $n$-alcanos.

\begin{tabular}{|l|l|l|l|l|l|}
\hline Composto & Curva analítica $\mathrm{Y}=\mathrm{ax}+\mathrm{b}$ & $\mathrm{Rec}$ & $\mathrm{r}^{2}$ & $\mathrm{LD}_{\mathrm{gg} \mathrm{kg}}$ & $\mathrm{LQ}^{-1} \mathrm{~kg}^{-1}$ \\
\hline Nonano & $\mathrm{Y}=48789,6 \mathrm{X}+(-7427,2)$ & & 0,985 & 4,3 & 6,3 \\
\hline Decano & $\mathrm{Y}=146906,2 \mathrm{X}+(-76417,2)$ & $36,4 \pm 11,8$ & 0,992 & 4,6 & 6,8 \\
\hline Undecano & $\mathrm{Y}=135900,1 \mathrm{X}+(-63425,2)$ & & 0,995 & 4,5 & 6,6 \\
\hline Dodecano & $\mathrm{Y}=140812,2 \mathrm{X}+(-49953,6)$ & $43,3 \pm 12,2$ & 0,996 & 2,2 & 3,3 \\
\hline Tridecano & $\mathrm{Y}=118134,7 \mathrm{X}+(1648,5)$ & & 0,989 & 1,5 & 2,2 \\
\hline Tetradecano & $\mathrm{Y}=114951,5 \mathrm{X}+(-250,1)$ & $68,5 \pm 4,9$ & 0,989 & 1,6 & 2,3 \\
\hline Pentadecano & $\mathrm{Y}=102177,6 \mathrm{X}+(2327,8)$ & & 0,987 & 1,7 & 2,5 \\
\hline Hexadecano & $\mathrm{Y}=82577,2 \mathrm{X}+(1807,0)$ & $66,3 \pm 7,2$ & 0,989 & 2,1 & 3,1 \\
\hline Heptadecano & $\mathrm{Y}=77701,1 \mathrm{X}+(1096,0)$ & & 0,988 & 2,2 & 3,3 \\
\hline
\end{tabular}




\begin{tabular}{|c|c|c|c|c|c|}
\hline Octadecano & $Y=79413,8 X+(1754,3)$ & $71,4 \pm 8,9$ & 0,987 & 2,2 & 3,2 \\
\hline Nonadecano & $Y=89268,8 X+(-414,8)$ & & 0,989 & 2,0 & 2,9 \\
\hline Eicosano & $\mathrm{Y}=93401,2 \mathrm{X}+(-158,2)$ & $75,7 \pm 8,2$ & 0,988 & 1,9 & 2,7 \\
\hline Heneicosano & $Y=98467,7 X+(-1551,3)$ & & 0,987 & 1,8 & 2,7 \\
\hline Docosano & $Y=102005,2 X+(-1392,4)$ & $78,2 \pm 8,1$ & 0,987 & 1,8 & 2,6 \\
\hline Tricosano & $\mathrm{Y}=157466,9 \mathrm{X}+(-39730,4)$ & & 0,998 & 1,4 & 2,1 \\
\hline Tetracosano & $Y=151996,2 X+(-38529,1$ & $82,6 \pm 8,5$ & 0,998 & 1,5 & 2,2 \\
\hline Pentacosano & $Y=153930,6 X+(-39092,6)$ & & 0,998 & 1,5 & 2,2 \\
\hline Hexacosano & $\mathrm{Y}=152475,3 \mathrm{X}+(-37672,1)$ & $81,3 \pm 9,1$ & 0,998 & 1,5 & 2,2 \\
\hline Heptacosano & $\mathrm{Y}=149579,3 \mathrm{X}+(-37839,9)$ & & 0,998 & 1,5 & 2,2 \\
\hline Octacosano & $\mathrm{Y}=146434,0 \mathrm{X}+(-35771,9)$ & $87,5 \pm 6,9$ & 0,998 & 1,5 & 2,2 \\
\hline Nonacosano & $\mathrm{Y}=140692,0 \mathrm{X}+(-34289,0)$ & & 0,998 & 1,6 & 2,4 \\
\hline Triacontano & $\mathrm{Y}=136802,5 \mathrm{X}+(-33712,3)$ & $88,4 \pm 7,1$ & 0,998 & 1,6 & 2,4 \\
\hline Untriacontano & $Y=137879,0 X+(-34767,9)$ & & 0,998 & 1,6 & 2,4 \\
\hline Dotriacontano & $\mathrm{Y}=137040,9 \mathrm{X}+(-35146,8$ & $83,3 \pm 10,1$ & 0,998 & 1,7 & 2,5 \\
\hline Tritriacontano & $\mathrm{Y}=126776,7 \mathrm{X}+(-30587,9)$ & & 0,998 & 1,8 & 2,6 \\
\hline Tetratriacontano & $Y=128759,7 x+(-34621,5)$ & $88,8 \pm 8,1$ & 0998 & 1,8 & 2,7 \\
\hline Pentatriacontano & $Y=127663,7 X+(-34968,4)$ & & 0,999 & 1,8 & 2,7 \\
\hline Hexatriacontano & $\mathrm{Y}=129108,5 \mathrm{X}+(-41434,8)$ & $85,4 \pm 9,5$ & 0,997 & 2,1 & 3,1 \\
\hline Heptatriacontano & $\mathrm{Y}=117744,8 \mathrm{X}+(-40326,4)$ & & 0,997 & 2,4 & 3,6 \\
\hline Octatriacontano & $Y=124229,9 X+(-57815,2)$ & $80,7 \pm 12,2$ & 0,991 & 4,9 & 7,3 \\
\hline Nonatriacontano & $Y=112339,2 X+(-59922,7)$ & & 0,993 & 5,9 & 8,7 \\
\hline Tetratriacontano & $Y=109345,3 X+(-69576,5)$ & $82,6 \pm 8,3$ & 0,993 & 6,2 & 9,0 \\
\hline
\end{tabular}

(a) coeficiente angular, (b) coeficiente linear, $\left(r^{2}\right)$ coeficiente de correlação, para a equação; (LD) limite de detecção; (LQ) limite de quantificação, REC: Recuperação \% \pm desvio padrão relativo expresso em \%,Resultados expressos em ( $\mu \mathrm{g}$ kg-1).
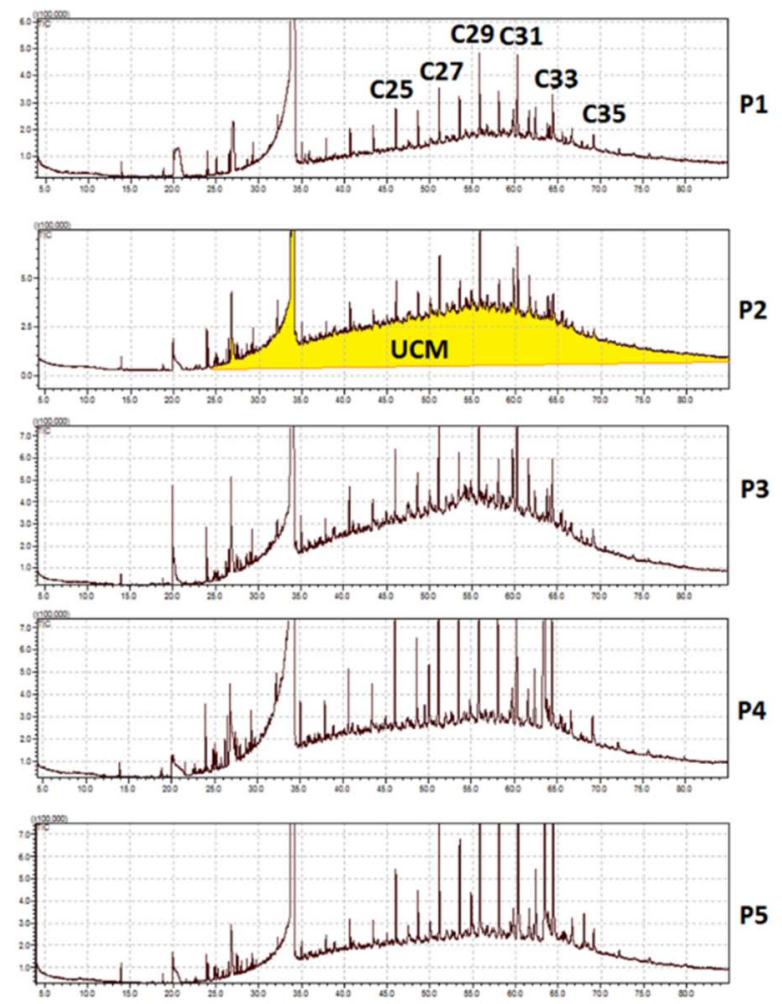

Figura 2: Cromatograma da fração F1 para os 5 pontos amostrados.

Podemos observar na Figura 2 que os cromatogramas da fração 1 de todos os pontos apresentaram um perfil cromatografico semelhante, com predominância de n-alcanos entre C23 a C35 acompanhadas de uma MCNR. Foram quantificados n-alcanos entre C16 e C 39.0 Nn-alcanos variaram de 1850,9 a 7754,0 $\mu \mathrm{g}$ $\mathrm{kg}^{-1}$ valores semelhantes aos encontrados na zona de manguezal (BERNARD et al., 1996)que apresentam sedimentos ricos em matéria orgânica. O ponto 4 apresentou os maiores níveis para $\sum n$-alcanos e MCNR seguido pelo ponto 2, resultados que estão em acordo com a caracterização preliminar. 
Tabela 3: Concentração de $n$-alcanos e MCNR ( $\mu \mathrm{kg}^{-1} \pm \mathrm{RSD} \%$ ) em nos pontos de amostragem do canal de drenagem pluvial no prolongamento da Av. São Francisco de Paula.

\begin{tabular}{|c|c|c|c|c|c|}
\hline Compostos & p1 & $\mathrm{p} 2$ & p3 & $\mathrm{p} 4$ & p5 \\
\hline Nonano & ND & ND & ND & ND & ND \\
\hline Decano & ND & ND & + & ND & ND \\
\hline Undecano & ND & ND & ND & ND & ND \\
\hline Dodecano & ND & ND & + & ND & + \\
\hline Tridecano & + & ND & + & + & + \\
\hline Tetradecano & + & ND & + & + & + \\
\hline Pentadecano & + & ND & + & + & + \\
\hline Hexadecano & + & ND & $8,7 \pm 12,7$ & + & + \\
\hline Heptadecano & $39,8 \pm 7,6$ & $103,2 \pm 34,1$ & $103,2 \pm 12,9$ & $133,3 \pm 13,9$ & $50,2 \pm 14,2$ \\
\hline Octadecano & $56,6 \pm 13,7$ & $81,0 \pm 14,6$ & $57,6 \pm 13,8$ & $126,6 \pm 10,1$ & $32,9 \pm 12,9$ \\
\hline Nonadecano & $58,6 \pm 12,7$ & $91,0 \pm 13,6$ & $63,0 \pm 12,8$ & $114,9 \pm 7,8$ & $31,7 \pm 14,9$ \\
\hline Eicosano & $37,8 \pm 14,9$ & $33,3 \pm 16,1$ & $49,0 \pm 1,9$ & $62,8 \pm 7,5$ & $29,0 \pm 15,2$ \\
\hline Heneicosano & $39,8 \pm 7,6$ & $81,0 \pm 14,6$ & $77,8 \pm 5,2$ & $109,0 \pm 12,7$ & $44,9 \pm 9,6$ \\
\hline Docosano & $58,7 \pm 6,0$ & $77,1 \pm 8,0$ & $60,4 \pm 6,0$ & $99,1 \pm 0,1$ & $44,9 \pm 9,6$ \\
\hline Tricosano & $77,6 \pm 14,5$ & $99,3 \pm 6,1$ & $89,2 \pm 8,4$ & $139,4 \pm 3,7$ & $60,7 \pm 9,7$ \\
\hline Tetracosano & $89,1 \pm 7,7$ & $88,2 \pm 6,6$ & $63,0 \pm 12,8$ & $135,4 \pm 3,3$ & $55,4 \pm 13,3$ \\
\hline Pentacosano & $112,3 \pm 6,3$ & $161,9 \pm 14,6$ & $149,4 \pm 18,2$ & $272,5 \pm 12,7$ & $126,8 \pm 11,3$ \\
\hline Hexacosano & $102,8 \pm 14,3$ & $110,3 \pm 5,4$ & $91,9 \pm 5,3$ & $214,7 \pm 10,9$ & $95,0 \pm 11,7$ \\
\hline Heptacosano & $126,8 \pm 13,3$ & $173,0 \pm 7,0$ & $187,1 \pm 8,1$ & $540,0 \pm 1,4$ & $214,0 \pm 13,5$ \\
\hline Octacosano & $109,2 \pm 14,6$ & $121,4 \pm 4,7$ & $100,6 \pm 4,6$ & $345,2 \pm 7,8$ & $158,5 \pm 13,0$ \\
\hline Nonacosano & $163,3 \pm 9,2$ & $242,9 \pm 9,8$ & $258,7 \pm 14,9$ & $2092,4 \pm 5,3$ & $272,5 \pm 11,2$ \\
\hline Triacontano & $99,2 \pm 13,9$ & $132,5 \pm 4,5$ & $89,2 \pm 0,9$ & $382,5 \pm 8,2$ & $169,1 \pm 14,2$ \\
\hline Untriacontano & $173,4 \pm 11,2$ & $257,3 \pm 4,8$ & $229,9 \pm 15,1$ & $1022,8 \pm 9,8$ & $296,2 \pm 3,2$ \\
\hline Dotriacontano & $89,7 \pm 8,4$ & $110,3 \pm 15,6$ & $71,8 \pm 2,2$ & $257,8 \pm 12,9$ & $132,0 \pm 6,2$ \\
\hline Tritriacontano & $97,9 \pm 12,2$ & $150,8 \pm 7,5$ & $129,4 \pm 11,8$ & $513,3 \pm 11,7$ & $158,0 \pm 9,9$ \\
\hline Tetratriacontano & $51,3 \pm 9,8$ & $69,8 \pm 16,4$ & $43,0 \pm 11,6$ & $162,2 \pm 13,6$ & $66,0 \pm 8,4$ \\
\hline Pentatriacontano & $60,7 \pm 12,1$ & $81,0 \pm 14,6$ & $80,4 \pm 9,4$ & $244,3 \pm 14,4$ & $84,5 \pm 14,0$ \\
\hline Hexatriacontano & $49,2 \pm 12,4$ & $58,8 \pm 1,8$ & $49,0 \pm 1,9$ & $139,0 \pm 5,0$ & $58,0 \pm 6,8$ \\
\hline Heptatriacontano & $53,3 \pm 12,6$ & $69,4 \pm 1,3$ & $57,7 \pm 3,5$ & $144,5 \pm 3,6$ & $60,7 \pm 6,9$ \\
\hline Octatriacontano & $58,7 \pm 14,7$ & $81,0 \pm 1,0$ & $57,7 \pm 3,5$ & $157,2 \pm 5,0$ & $60,7 \pm 6,9$ \\
\hline Nonatriacontano & $47,2 \pm 8,2$ & ND & $77,6 \pm 6,9$ & $287,4 \pm 13,3$ & $73,7 \pm 7,4$ \\
\hline Tetratriacontano & ND & ND & ND & $57,7 \pm 7,3$ & ND \\
\hline IPC & 1,4 & 1,7 & 2,3 & 3,2 & 1,7 \\
\hline RTA & 5,4 & 4,5 & 4,2 & 7,4 & 13,3 \\
\hline En-alcanos & 1850,9 & 2464,3 & 2245,1 & 7754,0 & 2374,6 \\
\hline MCNR & $50561,7 \pm 5,9$ & $163886,7 \pm 5,4$ & $106895,7 \pm 7,6$ & $172179,4 \pm 1,2$ & $90076,0 \pm 1,2$ \\
\hline MCNR / $\Sigma$ n-alcanos & 27,3 & 66,5 & 47,6 & 22,4 & 37,9 \\
\hline
\end{tabular}

IPC:índice preferencial de carbono, RTA: Razâo Terrígeno/Aquática, $\Sigma n$-alcanos: Somatória de n-alcanos , MCNR:mistura complexa não resolvidas

No ponto 1,3,4,5 foram detectados n-alcanos entre C13 e C16. O ponto1 está perto de uma grande avenida pavimentada com tráfego de veículos, ao lado de um estacionamento de um shopping center e ao lado de um posto de gasolina. A maior granulometria neste ponto (menos conteúdo de finos) indica um ponto de grande turbulência que impede a deposição, destes compostos. Os valores de IPC de 1,7 e a razão MCNR/乏 n-alcanos de 27,3 sugerem aportes recentes de HC de origem petrogênica. No ponto 2 a presença de nalcanos entre C20 e C24 sem a predominância ímpar/par, nesta faixa, sugere fonte petrogênicas. Podemos verificar o aumento na concentração de n-alcanos totais e da MCNR em relação ao ponto anterior. $\mathrm{O}$ alto valor da relação $M C N R / \sum$ n-alcanos $(66,5)$ em relação a outros pontos é um sinal de degradação desses contaminantes indicando contaminação crônica. Compostos mais leves, como C10, C12 a C15, aparecem no ponto 3, podendo ser quantificados $\mathrm{HCs}$ entre $\mathrm{C} 16$ e C39. Isso sugere uma nova entrada de contaminantes no canal de drenagem. No ponto 4 os n-alcanos de C13 a C16 foram detectados e quantificados entre $\mathrm{C} 17 \mathrm{e}$ C40. O valor de 22,4 para a relação MCNR/ $\Sigma$ n-alcanos indica a entrada recente de tais hidrocarbonetos. 
Como descrito anteriormente neste ponto ocorre a entrada de um segundo canal de drenagem pluvial, adicionando a carga de poluentes transportada por este ao canal estudado.

Razões MCNR/ $n$-alcanos variaram entre 22,4 e 66,5 ao longo do canal, segundo Tolosa et al. (2004) valores superiores a 10 indicam uma contaminação crônica por hidrocarbonetos de origem petrogênica o que é confirmado pelas localização da MCNR entre nC18 e nC35 (BOULOUBASSI et al., 1993). O ponto 5 é um ponto de encontro com outro canal de drenagem que atravessa uma área rural com baixas contribuições antropogênicas, reflete no declínio na concentração de Hidrocarbonetos, alifáticos, HTP e $\sum n$-alcanos. 0 valor de $\Sigma n$-alcanos neste estudo estão de acordo com os níveis determinados em outros ambientes hídricos, como os descritos na Baia de Ushuaia Bay, Tierra del Fuego - Patagonia, Argentina de 280 a $11720 \mathrm{ug} \mathrm{kg}^{-1}$, (COMMENDATORE et al., 2012) e os descritos por Sanches Filho et al. (2017) no Canal São Gonçalo- Pelotas -Brasil entre 854,3 e 199433,9 $\mathrm{g} \mathrm{kg}^{-1}$.

O índice preferencial de carbono (IPC), variou de 1,4 a 3,2 (Tabela 2). Valores de IPC, superior a 1, indicam a predominância de número ímpar de átomos de carbono nas moléculas de hidrocarbonetos. IPCs entre 4 e 7 indicam $n$-alcanos de origem biogênicos, e valores próximos a 1 indicam contaminação petrogênica ,valores intermediários entre 1 e 3 indicam ambas entradas biogênicas e de combustíveis fósseis (BOULOUBASSI et al., 2001; MEDEIROS et al., 2004; SANCHES FILHO et al., 2013). Podemos observar que o valor de IPC aumenta em direção ao fluxo do canal entre os pontos 1 e 4 e diminui no ponto 5 indicando entradas de HCs tanto de origem biogênica como Petrogênica ao longo do canal.

A razão terrígena/aquática (RTA) que variou entre 4,2 e 13,3, avalia a importância da presença de hidrocarbonetos de origem de plantas aquática e/ou terrestres, pois quanto maiores estes valores, maior a colaboração de plantas superiores terrestres. Todos os pontos apontam para os HCs de origem biogênica relacionados a plantas superiores, o que pode ser observado na figura 2 pelo predomínio de $n$-alcanos ímpares entre C23 e C35.

\section{CONCLUSÕES}

Os níveis de hidrocarbonetos (HTP, alifáticos e $n$-alcanos) foram considerados altos, confirmando o impacto do runoff urbano coletado e transportado pelo sistema de drenagem pluvial, sobre os recursos hídricos. Os resultado apontaram para HCs tanto de origem petrogênica como biogênica indicando uma origem complexa da matéria orgânica descrita e acumulada nos sedimentos. A questão é de extrema importância uma vez que a maioria das cidades não tratam as águas do sistema de drenagem pluvial, e os descarregam diretamente em lagos, rios e áreas estuarinas.

AGRADECIMENTOS: Os autores agradecem ao CNPq, FAPERGS e FINEP por seu apoio financeiro.

\section{REFERÊNCIAS}

ANDERSSON, M.; KLUG, M.; EGGEN, O. A.; OTTESEN, R. T.. Polycyclic aromatic hydrocarbons (HPA's) in sediments from lake Lille Lungegardsvannet in Bergen, western Norway; appraising pollution sources from the urban history. Science of the Total Environment, v.470-471, p.1160-1172, 2014. DOI: https://doi.org/10.1016/j.scitotenv.2013.10.086 
APRILE, F. M.; BIANCHINI JR, I.. Adequação metodológica para determinação do nitrogênio orgânico total em macrófitas aquáticas e sedimentos. Revista Brasileira de Biociências, v.8, p.49-56, 2003.

AZEVEDO, D. A.; SANTOS, C. Y. M.; AQUINO NETO, F. R. Identification and seasonal variation of atmospheric organic pollutants in Campos dos Goytacazes, Brazil. Atmospheric Environment, v.36, n.14, p.2383-2395, 2002. DOI: https://doi.org/10.1016/S1352-2310(01)00585-4

BARBOSA, A. E.; FERNANDES, J. N.; DAVID, L. M.. Key issues for sustainable urban stormwater management. Water Research, v.46, n.20, p.6787-6798, 2012. DOI: https://doi.org/10.1016/j.watres.2012.05.029

BERNARD, D.;PASCALINE, H.; JEREMIE, J. J.. Distribution and origin of hydrocarbons in sediments from lagoons with fringing mangrove communities. Marine Pollution Bulletin, v.32, n.10, p.734-739, 1996. DOI:

https://doi.org/10.1016/0025-326X(96)00034-3

BISSUTTI, I.; HILKE, I.; RAESSLER, M.. Determination of total organic carbon-an overview of current methods. Trends in Analytical Chemistry, v.23, n.10-11, p.716-726, 2004. DOI: https://doi.org/10.1016/j.trac.2004.09.003

BOULOUBASSI, I.; FILLAUX, J.; SALIOT, A.. Hydrocarbons in surface sediments from the Changjiang (Yangtze River) Estuary, East China Sea. Marine Pollution Bulletin, v.42, n.12, p.1335-1346, 2001. DOI:

https://doi.org/10.1016/S0025-326X(01)00149-7

CELINO, J. J.; TRIGÜIS, J. A.; VEIGA, I. G.; QUEIROZ, A. F. D. S. Biomarcadores e 'fingerprints' de hidrocarbonetos nos sedimentos de manguezais na porção norte da Baía de Todos os Santos. Revista Brasileira de Geociências, v.38, n.2, p.186-196, 2008.

COLOMBO, J. C.; PELLETIER, C. H.; BROCHU, A.; KHALIL, M.; CATOGGIO, J. A.. Determination of hydrocarbon sources using n-alkane and polyaromatic hydrocarbons distribution indexes.Case study: Rio de La Plata Estuary, Argentina.

Environmental Science \& Technology, v.23, p.888-894, 1989. DOI: https://10.1021/es00065a019

COMMENDATORE, M. G.; NIEVAS, M. L.; AMIN, O.; ESTEVES, J. L.. Sources and distribuition of aliphatic and polyaromatic hydrocarbons in coastal sediments from the Ushuaia Bay (Tierra del Fuego, Patagonia, Argentina). Marine Environmental Research, v.74, p.20-31, 2012. DOI: https://10.1016/j.marenvres.2011.11.010

ERIKSSON, E.; BAUN, A.; SCHOLES, L.; LEDIN, A.; AHLMAN, S.; REVITT, M.. Selected stormwater priority pollutants: A European perspective. Environmental Science \& Technology, v.383, n.1-3, p.41-51, 2007. DOI: https://doi.org/10.1016/j.scitotenv.2007.05.028

GRIMMLER, M. U.; BETEMPS, G. R.; FERREIRA, M. M.; SANCHES FILHO, P. J.. Determinação de metais tóxicos em sedimento de canal de drenagem pluvial na zona urbana. Revista Ibero-Americana de Ciências Ambientais, v.7, n.1, p.201-209, 2016. DOI: http://doi.org/10.6008/SPC2179$\underline{6858.2016 .001 .0017}$
GNECCO, I.; BERRETTA, C.; LANZA, L. G.; LA BARBERA, P.. Storm water pollution in the urban environment of Genoa, Italy. Atmospheric Environment, v.77, n.1-4, p.60-73, 2005. DOI: https://doi.org/10.1016/j.atmosres.2004.10.017

GÖBEL, P.; DIERKES, C.; COLDEWEY, W. G.. Storm water runoff concentration matrix for urban areas. Journal of Contaminant Hydrology, v.91, n.1-2, p.26-42, 2007. DOI: https://doi.org/10.1016/j.jconhyd.2006.08.008

HOWITT, J. A.; MONDON, J.; MITCHELL, B. D.; KIDD, T.; ESHELMANA, B.. Urban stormwater inputs to an adapted coastal wetland: Role in water treatment and impacts on wetland biota. Science of the Total Environment, v.485486, p.534-544, 2014. DOI:

https://doi.org/10.1016/j.scitotenv.2014.03.101

IUPAC. Compendium of Chemical Terminology. 2 ed. 1997.

LI, Y. T.; LI, F. B.; CHEN, J. J.; YANG, G.Y.; WAN, H. F.; ZHANG, T. B.; ZENG, X. D.; LIU, J. M.. The concentrations, distribution and sources of HPA's in agricultural soils and vegetables from Shunde, Guangdong, China, Environ. Environmental Monitoring and Assessment, v.139, p.61-76, 2008. DOI: https://10.1007/s10661-007-9816-x

MANNINO, M. R.; ORECCHIO, S.. Polycyclic aromatic hydrocarbons (HPA's) in indoor dust matter of Palermo (Italy) area extraction, GC-MS analysis, distribution and sources. Atmospheric Environment, v.42, p.1801-1817, 2008. DOI: https://doi.org/10.1016/j.atmosenv.2007.11.031

MARTINS, C. C.; BÍCEGO, M. C.; TANIGUCHI, S.; MONTONE, R. C.. Aliphatic and polycyclic aromatic hydrocarbons in surface sediments in Admiralty Bay, Kin George Island, Antarctica, Antarctic Science, v.16, p.117-122, 2004. DOI: https://doi.org/10.1017/S0954102004001932

MARTINEZ, E.; GROS, M.; LACORTE, S.; BARCELÓ, D. Simplified procedures for the analysis of polycyclic aromatic hydrocarbons in water, sediments and mussels. J. Chromatografe, v.1047, n.2, p.181-188, 2004. DOI: https://doi.org/10.1016/j.chroma.2004.07.003

MEDEIROS, P. M.; BICEGO, M. C.. Investigation of natural and anthropogenichydrocarbon inputs in sediments using geochemical markers. I. Sao Sebastiao/SP/Brazil. Marine Pollution Bulletin, v.49, n.11-12, p.761-769, 2004. DOI: https://10.1016/i.marpolbul.2004.06.002

MILLE, G.; ASIA, L.; GUILIANO, M.; MALLERET, L.; DOUMENQ, $P$.. Hydrocarbons in coastal sediments from the Mediterranean Sea (Gulf of Fos area, France) Marine Pollution Bulletin, v.54, n.5, p.566-574, 2007. DOI: https://doi.org/10.1016/j.marpolbul.2006.12.009

NISHIGIMA, F. N.; WEBER, R. R.; BíCEGO, M. C.. Aliphatic and aromatic hydrocarbons in sediments of Santos and Cananéia/SP/Brazil. Marine Pollution Bulletin, v.42, n.1, p.1064-1072, 2001. DOI: https://doi.org/10.1016/S0025326X(01)00072-8

SAITO, Y.; NISHIMURA, A.; MATSUMOTO, E.. Transgressive Sand sheet covering the shelf and upper slope off sendal, northeast Japan. Marine Geology, v.89, n.3-4, p.249-258, 1989. DOI: https://doi.org/10.1016/0025-3227(89)90078-9 
SANCHES FILHO, P. J.; BÖHM, E. M.; BÖHM, G. M. B.; MONTENEGRO, G. O.; SILVEIRA, A. L.; BETEMPS, G. R.. Determination of hydrocarbons transported by urban runoff in sediments of São Gonçalo Channel (Pelotas/RS/Brazil). Marine Pollution Bulletin, v.114, n.2, p.1088-1095, 2017. DOI: http://dx.doi.org/10.1016/j.marpolbul.2016.10.024

SANCHES FILHO, P. J.; LUZ, L. P.; BETEMPS, G. R.; CARAMÃO, E. B.. Evaluation of surface sediment contamination by polycyclic aromatic hydrocarbons in the 'Saco do Laranjal' (Patos Lagoon, Brazil). Marine Pollution Bulletin, v.64, n.9, p.1933-1937, 2012. DOI:

https://doi.org/10.1016/j.marpolbul.2012.04.010

SANCHES FILHO, P. J.; LUZ, L. P.; BETEMPS, G. R.; SILVA, M. D. R. G.; CARAMÃO E. B.. Studies of n-alkanes in the sediments of Colony Z3 (Pelotas - RS - Brazil). Braz. J. Aquatic Science and Technology, v.17, n.1, p.27-33, 2013. DOI: http://dx.doi.org/10.14210/bjast.v17n1.p27-33

SIMPSON, M. J.; CHEFETZ, B.; DESHMUKH, A. P.; HATCHER, P. G.. Comparison of polycyclic aromatic hydrocarbon distributions and sedimentary organic matter characteristics in contaminated, coastal sediments from Pensacola Bay, Florida. Marine Environmental Research, v.59, n.2, p.139163, 2005. DOI: https://doi.org/10.1016/j.marenvres.2003.09.003

SUGUIO, K.; PETRI, S.. Stratigraphy of the Iguape-Cananéia lagoonal region sedimentary deposits, São Paulo State, Brazil. Part I: Field observations and grain size analysis. Boletim Ig Instituto de Geociências USP, São Paulo, v.4, p.120, 1973. DOI: https://doi.org/10.11606/issn.23168978.v4i0p01-20

TEDESCO, M. J.; GIANELLO, C.; BISSANI, C. A.; BOHNEN, H.; EVOLKWEISS, S. J.. Análises de solo, plantas e outros materiais: Boletim técnico n⒌ 2 ed. Universidade Federal do Rio Grande do Sul, 1995.

TOLOSA, I.; MORA, S.; SHEIKHOLESLAMI, M. R.; VILLENEUVE, J. P.; BARTOCCI, J.; CATTINI, C.. Aliphatic and aromatic hydrocarbons in coastal Caspian Sea sediments. Marine
Pollution Bulletin, v.48, n.3, p.44-60, 2004. DOI:

https://doi.org/10.1016/S0025-326X(03)00255-8

UNEP. United Nations Environment Programme.

Determination of petroleum hydrocarbons in sediments:

References Methods for Marine Pollution studies. Nairóbi: UNEP, 1992.

VIÑAS, L.; FRANCO, M. A.; GONZÁLEZ, J. J.. Polycyclic aromatic hydrocarbon composition of sediments in the Río de Vigo (NW Spain), Arch. Archives of Environmental Contamination and Toxicology, v.57, p.42-49, 2009.

VOLKMAN, J. K.; JOHNS, R. B.; GILLAN, F. T.; PERRY, G. J.. Microbial lipids of an intertidal sediments. I - Fatty acids and hydrocarbons. Geochimica et Cosmochimica Acta, v.44, n.80, p.1133-1143, 1980. DOI: https://doi.org/10.1016/0016-7037(80)90067-8

VOLKMAN, J. K.; HOSTETTLER, F. D.; NEIL, G.; BAYOR, H. Identification of natural, anthropogenic and petroleum hydrocarbons in aquatic sediments, Science of the Total Environment, v.112, n.92, p.203-219, 1992. DOI: https://doi.org/10.1016/0048-9697(92)90188-X

WU, Y.; ZHANG, J. Z.; MI, T.; LI, B.. Occurrence of n-alkanes and polycyclic aromatic hydrocarbons in the core sediments of the Yellow Sea. Marine Chemistry, v.76, n.1, p.1-15, 2001. DOI: https://doi.org/10.1016/S0304-4203(01)00040-8

YUNKER, M. B.; MACDONALD, R.W.; CRETNEY, W. J.; FOWLER, B. R.; MCLAUGHLIN, F. A.. Alkane, terpene, and polycyclic aromatic hydrocarbon geochemistry of the Mackenzie River and Mackenzie Shelf: Riverine contributions to Beaufort Sea coastal sediment. Geochimica et Cosmochimica Acta, v.57, n.13, p.3041-3061,1993. DOI: https://doi.org/10.1016/0016-7037(93)90292-5

ZHENG, G. J.; RICHARDSON, B. J.. Petroleum hydrocarbons and polycyclic aromatic hydrocarbons (HPA's) in Hong Kong marine sediments, Chemosphere, v.38, n.11, p.2625-2632, 1999. DOI: https://doi.org/10.1016/S0045-6535(98)00470-6

A CBPC - Companhia Brasileira de Produção Científica (CNPJ: 11.221.422/0001-03) detém os direitos materiais desta publicação. Os direitos referem-se à publicação do trabalho em qualquer parte do mundo, incluindo os direitos às renovaç̃ões, expansões e disseminações da contribuiç̃o, bem como outros direitos subsidiários. Todos os trabalhos publicados eletronicamente poderão posteriormente ser publicados em coletâneas impressas sob coordenação da Sustenere Publishing, da Companhia Brasileira de Produção Científica e seus parceiros autorizados. Os (as) autores (as) preservam os direitos autorais, mas não têm permissão para a publicação da contribuição em outro meio, impresso ou digital, em português ou em tradução. 